\title{
City Design and the Transmission of COVID-19
}

\author{
Mark Stevenson, Jason Thompson, Branislava Godic and Thanh Ho
}

\begin{abstract}
We outline recent research in which Google maps were acquired for cities from across the globe. Using unique methods including convolutional neural networks and graph-based approaches, nine city designs were identified based on land-use characteristics such as road networks, public transit, green space and blue space. In this chapter, we assess the influence of these land-use characteristics in each of the city designs on the transmission of COVID-19 and hence the number of positive cases of COVID-19. The chapter highlights the role of city design with respect to the transmission of COVID-19 and particularly, on transmission of the virus in cities identified with high density road networks and public transit. The chapter concludes by discussing the potential policy implications arising from certain city designs and infectious disease out-breaks.
\end{abstract}

\subsection{The Pandemic that is COVID-19}

Between November and December 2019 numerous patients presented to hospitals in the city of Wuhan (Hubei Province, China) with severe respiratory symptoms due to a virial infection. By January 2020, officials in agencies such as the World Health Organisation were aware of the onset of what has become the COVID-19 pandemic. To complicate the onset of the epidemic, the observed infections coincided with the Chinese Lunar New Year holiday where an estimated 3 billion trips are undertaken during the month-long holiday period [1]. Movement of large populations, facilitated by China's established transport system, was a significant challenge during the early stages of the outbreak, just as it was one hundred years earlier where transmission of the Spanish Flu, was exacerbated via the various transport modes carrying returning soldiers from World War 1 [2]. China moved quickly to mitigate the spread of COVID-19 by closing-down its extensive transport system [3]. There is considerable evidence pointing to the value of such measures with Tian et al [4] estimating 37\% fewer cases of COVID-19 in cities in which the transport systems were closed-down.

The travel restriction on January 23, 2020, in Wuhan City and the following lock-down in the whole Hubei Province and nearby cities managed to slow the transmission of the virus in China. However, by January 31, 2020, an estimated 1.5 infected air travellers who had departed China, were transmitting the virus each day across the globe [5]. The hyper-mobility and interconnectedness of 21 st century transport networks, including high-speed and high-capacity rail and intercontinental air travel have enabled the virus to spread rapidly [6].

Over the ensuing 8 months, COVID-19 cases (or specifically SARS-CoV-2 infections) have been reported in every country other than a few island states such as Cayman Island, Mauritius, Guernsey, Fiji and Dominica [7]. As of 14 August 2020, there were more than 20.7 million cases of COVID-19 in more than 188 countries and territories, resulting in more than 750,000 deaths [7].

As observed in Figure 10.1, there is considerable country variation in the number of COVID-19 cases. One explanation for the variation between countries is the differences in government 


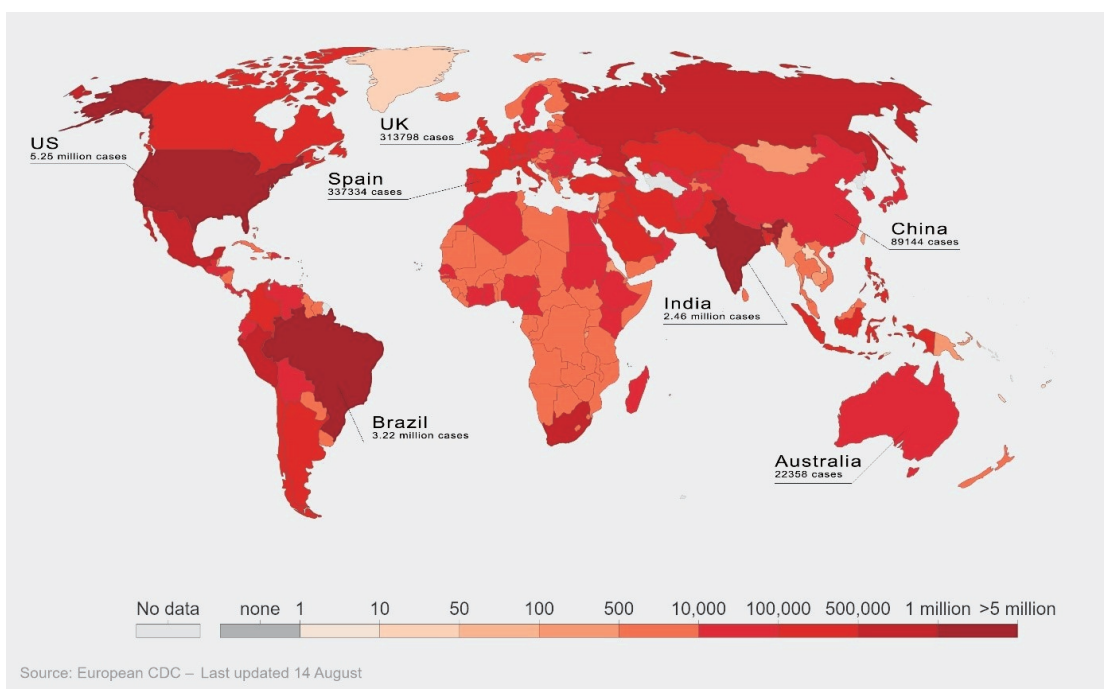

\section{FIGURE 10.1}

Global Variation in COVID-19 Cases

response to managing the pandemic. Recent research [8] has mapped a country's response to the pandemic by the timeliness to implement efficacious public health responses. Responses such as, the implementation of physical distancing strategies, remaining at home other than for essential purposes, ongoing enhancements of the health system, extensive travel restrictions, extensive contact-tracing and quarantining of infected individuals and social and economic provisions to assist individuals with the impact of closing-down much of a country's economy in an effort to minimise transmission. As observed in Figure 10.2, two months after the first notification of COVID-19, individual government response to the pending pandemic varied with countries reporting cases of COVID-19 implementing many of the public health responses described above whilst other countries being much slower to respond, presumably associated with no or few cases being reported. Responding quickly to the pandemic, however, did not confer a rapid reduction in infections. As highlighted by the dark brown colour in Figure 10.2, countries such as China, the United States of America (US) and Brazil instituted many public health responses by March 22, 2020 and at the time of writing (August 2020) both the US and Brazil have not suppressed the transmission of COVID-19. It should be noted, however, that implementation of 'stay at home' orders in countries such as the United States were not implemented on a country-wide level, but rather on a state or region-by-region basis that followed the progression of new COVID-19 cases across the country.

Not only is there is considerable variation in COVID-19 cases between countries there is also considerable variation between regions and cities within countries. Figure 10.3 highlights the considerable variation observed in COVID-19 cases within the US and the United Kingdom.

The variation within countries observed in Figures 10.3 is not easily explained as it likely reflects a multitude of factors ranging from governance structures, a poor safety culture within the country, lack of resources (including the ability to institute quarantine measures), inadequate health resources, and poor communication with the public, to name a few [9]. Importantly, the influence of a city's urban design and the variation of such designs between cities in a country may also be an important factor influencing the transmission of COVID-19. Only recently, has the research literature explored a number of these designs with respect to health outcomes (specifically road trauma) [10] and certainly not with respect to COVID-19.

In the remainder of this chapter we describe how city designs can be classified based on land-use characteristics such as road networks, public transit, green space and blue space (e.g., water bodies). Once a classification system is identified for specific city designs, we will assess whether specific city designs are associated with the reported cases of COVID-19. 


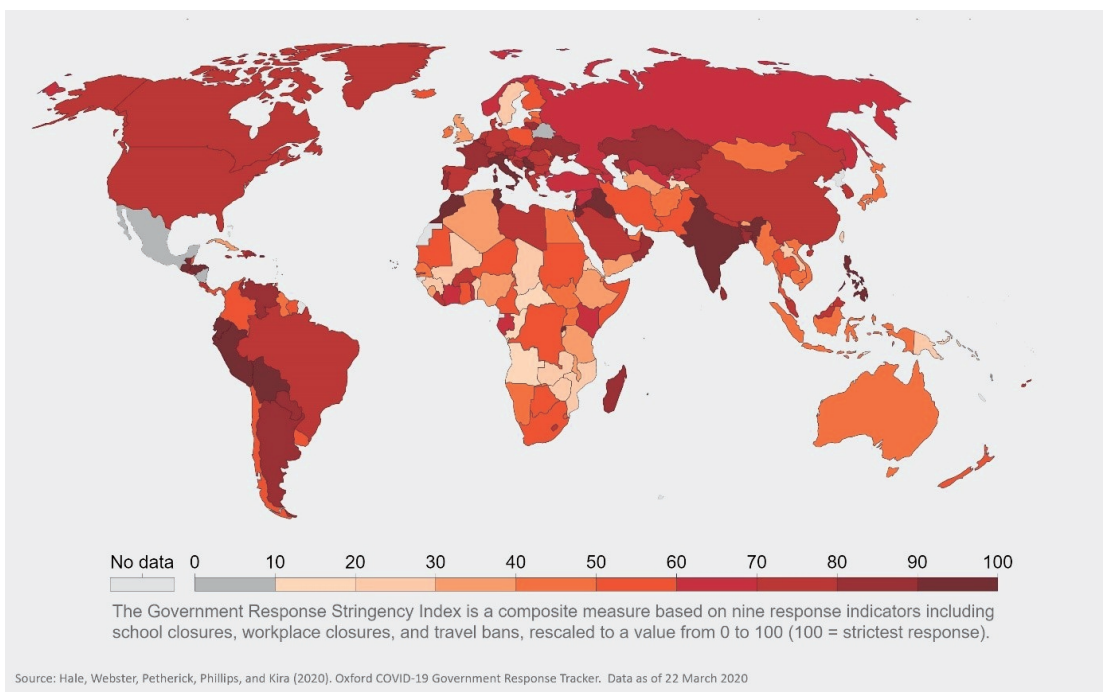

\section{FIGURE 10.2}

Country Variation in Government Response to COVID-19 as of March 22, 2020

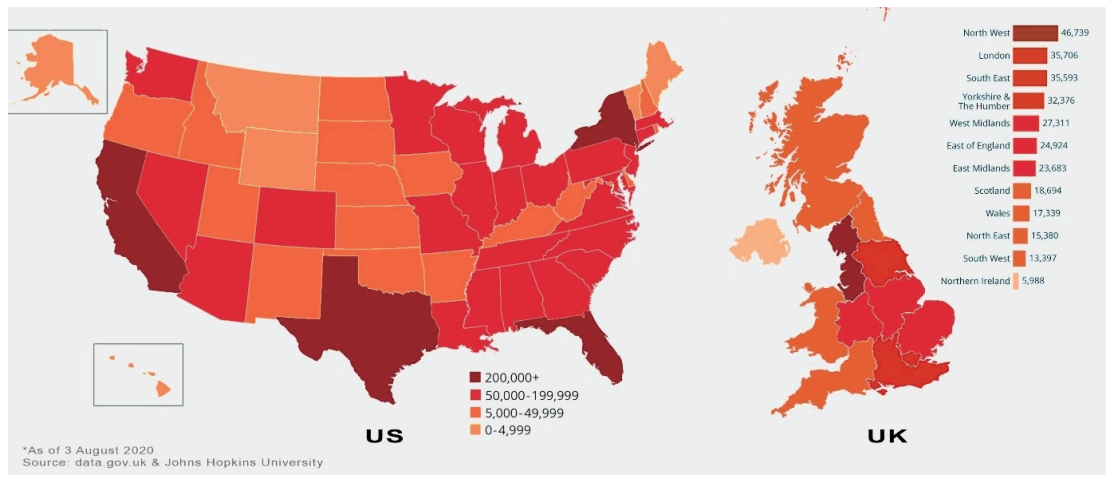

\section{FIGURE 10.3}

Variation in COVID-19 Cases in the US and the United Kingdom

\subsection{Using Spatial Data to Identify Global City Design}

To assess whether specific city design elements could be classified into discrete categories, we used the Google maps API to analyse a set of 1000 stylised maps for every city listed in the 2018 United Nations world population prospect report [11] with a population exceeding 300,000. A total of 1667 cities across the globe were identified. Using a convolutional neural network (CNN) modelling approach based on 'Inception V3' architecture [12], we identified whether cities could be correctly classified as being from their actual (ground truth) location. Each map was of approximately $400 \mathrm{~m}$ $\mathrm{x} 400 \mathrm{~m}$ size and contained representation of road networks, public transport networks, green space (e.g., parks and reserves) and blue space (water bodies). Details of the approach including the calibration of the stages are described in Thompson et al [10].

Once classification of cities to their ground truth location was completed, a graph of the confusion matrix produced by the CNN was created that represented nodes (cities) connected by vertices that represented cities occasionally mistaken for one another in the CNN process. A modularity analysis (similar to a cluster analysis) was then performed on the graph to isolate groups 
of cities among the 1667 that were regularly misclassified (i.e., confused for one another). This process produced a set of nine major city design types. Table 10.1 describes the nine city designs, a brief description of the design and lists the proportion of cities that fall within the respective city designs. The majority (64\%) of low- and middle-income countries and their respective cities, fall within the city design Informal and Irregular; city designs with informal road infrastructure and limited public transit. In contrast, many cities from high income countries were classified under city designs described as either the Motor City and or Intense city design characterised either by high capacity road networks or high-density road networks and high public transit (see Figure 10.4).

Clearly not all cities in a country have the same design. Figure 10.5 illustrates the variation in city design within a country. The proportion of city design types within a country are based on the results of the convolutional neural network analysis. It is evident there is considerable variation in city designs within some countries and absolutely no variation in other countries. For example, there is considerable variation among cities in Vietnam with 4 city designs identified and similarly in China. In contrast, cities within Sweden, Switzerland, Norway, Ireland and Denmark only fall within one city design namely, the High Transit city design, which reflects urban form that is medium density with high capacity formal road networks and high public transport.

The opportunity to classify cities using objective data from standardised maps highlights the utility of spatial data. Such data provides insights on urban form not previously reported. However, the classification of nine city designs is limited to the four land use characteristics that could be systematically attained from the google maps. Nonetheless, these characteristics are important elements particularly in the context of pandemics whereby highly integrated public transit systems can escalate the transmission of infectious diseases.

TABLE 10.1

Types of city design and the proportion of cities classified under each design

\begin{tabular}{llc}
\hline $\begin{array}{l}\text { Type of City } \\
\text { Design }\end{array}$ & Description & $\begin{array}{c}\text { Cities classified in } \\
\text { the design (\%, N) }\end{array}$ \\
\hline Informal & $\begin{array}{l}\text { Sparse, low capacity informal road infrastructure, limited rail } \\
\text { transport, low formal green space }\end{array}$ & $23 \%(365$ cities $)$ \\
\hline Irregular & $\begin{array}{l}\text { High green space, mixed formal and informal infrastructure, few } \\
\text { high capacity road networks, limited mass transit }\end{array}$ & $21 \%(311$ cities $)$ \\
\hline Large Blocks & $\begin{array}{l}\text { Medium density, formal low and high capacity road networks, } \\
\text { medium railed transport }\end{array}$ & $9 \%(146$ cities $)$ \\
\hline Cul-de-sac & $\begin{array}{l}\text { Very high density, low capacity mixed formal and informal road } \\
\text { networks, low mass transit. }\end{array}$ & $1 \%(26$ cities $)$ \\
\hline High-Transit & $\begin{array}{l}\text { Medium density, high capacity, formal road networks, high public } \\
\text { transport }\end{array}$ & $10 \%(163$ cities $)$ \\
\hline Motor city & $\begin{array}{l}\text { Medium to low density, high capacity, grid-based, road networks, } \\
\text { medium railed transport }\end{array}$ & $10 \%(158$ cities $)$ \\
\hline Chequerboard & $\begin{array}{l}\text { High density, medium capacity mixed formal and informal road } \\
\text { networks, medium public transport }\end{array}$ & $16 \%(257$ cities $)$ \\
\hline Intense & $\begin{array}{l}\text { Very high density, mixed formal high capacity and informal road } \\
\text { networks, high public transport }\end{array}$ & $1 \%(22$ cities $)$ \\
\hline Sparse & $\begin{array}{l}\text { Low capacity, low density formal and informal road networks, low } \\
\text { public transport }\end{array}$ & $9 \%(142$ cities $)$ \\
\hline
\end{tabular}




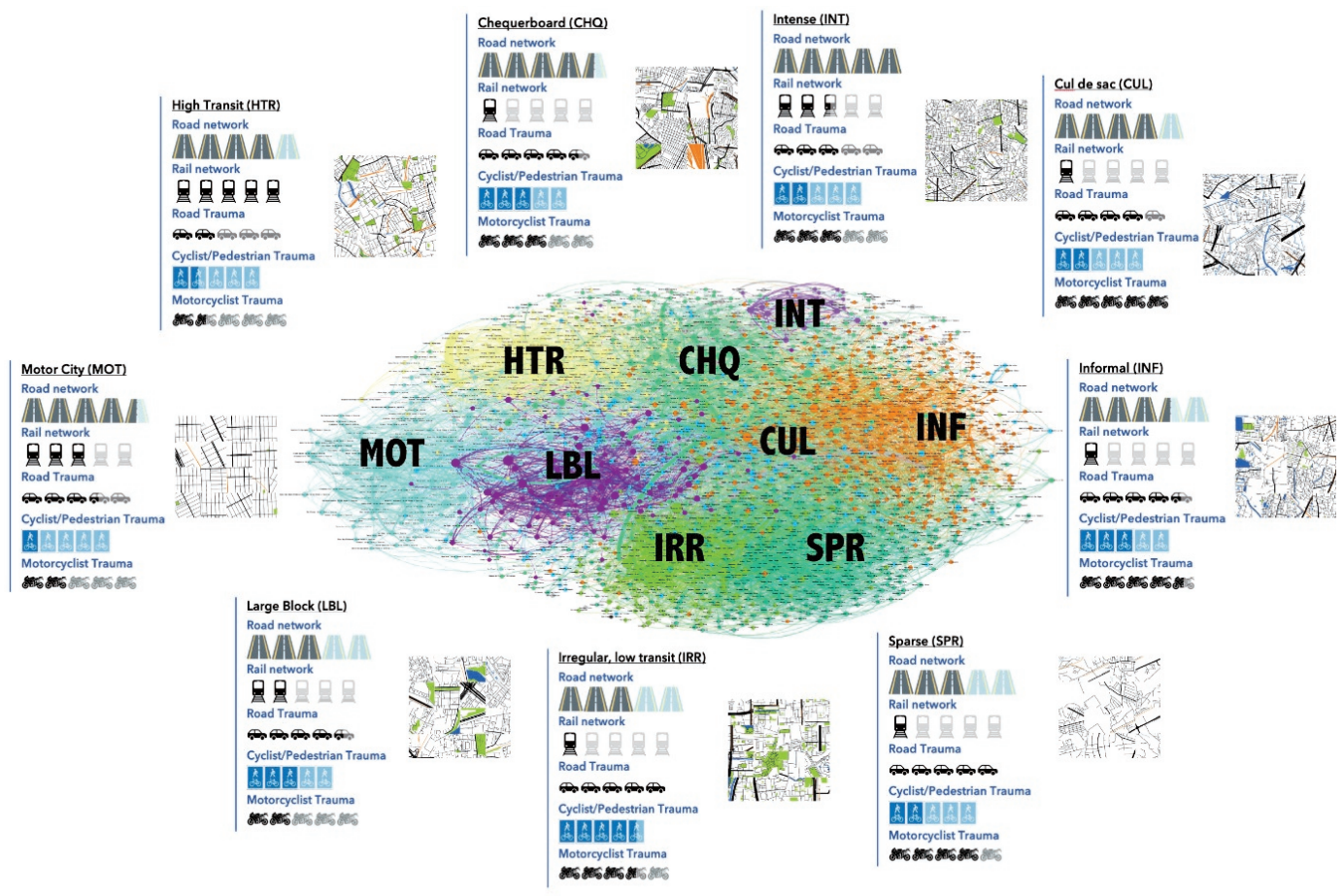

Figure showing each city design's road and public transport characteristics, sample map images and the relationship between each city type and road trauma outcomes.

\section{FIGURE 10.4}

Confusion Matrix Produced by the Convolutional Neural Network

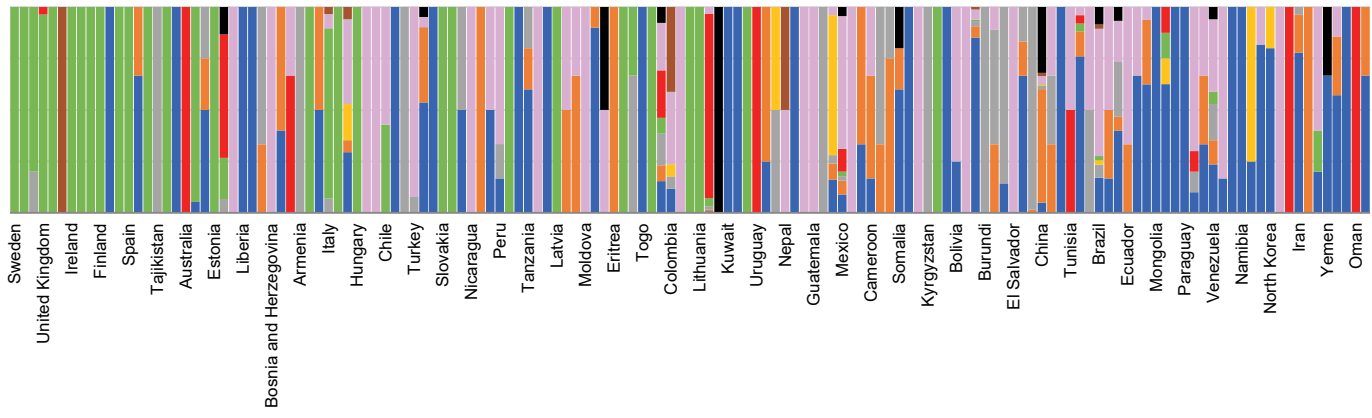

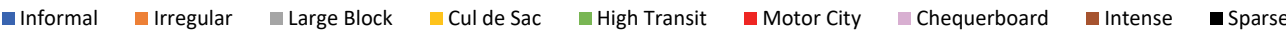

\section{FIGURE 10.5}

Proportions of city design types identified in each country 


\subsection{Relationship Between City Design and COVID-19}

As the COVID-19 pandemic enters its 9 month and many countries such as Australia are grappling with a second wave of outbreaks, important public health responses to mitigate the transmission particularly managing public transit and boarder control both between cities and countries are of increasing importance. Understanding which city designs (if any) are likely to increase the propensity to mitigate transmission is of utmost importance. Given the characteristics of the nine city designs, one might expect that cities that are categorised under the following designs as particularly vulnerable for increased transmission especially if the government response is not proactive namely; Large Block, High Transit, Chequerboard and Intense city designs; these designs have higher population densities along with greater density of road and public transit.

While the relationship of city designs that include reference to road and public transport networks bears a logical relationship to rates of road trauma across countries, the extent to which city design contributes to communicable disease is less certain. It could be hypothesised that a higher proportion of public transit infrastructure and consequent use might lead to greater likelihood of close contact between individuals and person-to-person transmission. Alternatively, the proportion of public transit may be less important than population density in the city, which could determine how close citizens may be forced together on any given day. Finally, it may be these factors are ultimately less important than the timing and intensity of public health and social policies (e.g., stay at home orders) that promote social distancing and limit the potential negative impact of features associated with compact city design - features we have increasingly come to associate with good rather than poor health [13].

To investigate the relationship between city design features and COVID-19, we took an available subset of cities and assessed, up to the time that initial 'stay at home' orders were implemented, an array of factors against the mean growth in COVID-19 cases for each city. We considered the following factors: i) the proportion of each city's land-area dedicated to railed public transit, ii) the proportion of each city's land-area dedicated to road networks, iii) the recorded population density of each city in persons per $\mathrm{km}^{2}$, iv) the mean block size of each city, v) the regularity of each city's blocks (e.g., how 'square' they are), and vi) the timing of implementation of initial 'stay-at home' orders for each city. This last factor is important to consider alongside the characteristics of city design in order to understand the growth (or decline) in COVID-19 cases under a relatively 'natural' or unmitigated scenario, before the implementation of stringent public health policy measures that might otherwise restrict the free movement and interaction of city residents.

A total of 220 cities from the original list of 1667 were selected. This was the total number of cities for which individual daily cases for COVID-19 data were available at the time of writing. Given the reduced number of cities available for analysis across city designs we restricted our analysis to the measured characteristics of images from within these cities as described above, rather than describing associations with city design types.

Across the selected cities, sixty-three cities were excluded due to missing data across one or more of the factors described above hence, a total of 157 cities were analysed. The findings from the analysis highlighted that a city's design across public transit infrastructure, road infrastructure, block size, and block regularity were not associated with the rates of COVID-19 case growth. Importantly, the findings pointed to a city's population density as significantly associated with case growth albeit, a negative relationship indicating that cities with higher population densities were associated with lower reported case growth from the time the initial case was reported to the first stay-at-home orders. Similarly, days taken between initial case identification and stay-at-home orders were also negatively associated with mean case growth over time.

Focusing on individual cities and regions included in this analysis provides some insight into why both population density and extended delays to the instigation of stay-at-home orders appear negatively associated with the cases of COVID-19. Many cities with high population density are located within countries that also had long delays between identification of their first COVID-19 case and the instigation of stay-at-home orders including those in Japan, Vietnam, Thailand and the city-state of Singapore (see Figure 10.6). This delay does not infer increased transmission as these countries have touted effective control of COVID-19 infections through means other than 
economic and stay-at-home orders including mass testing, mask-wearing, hygiene promotion, and rapid contact tracing.
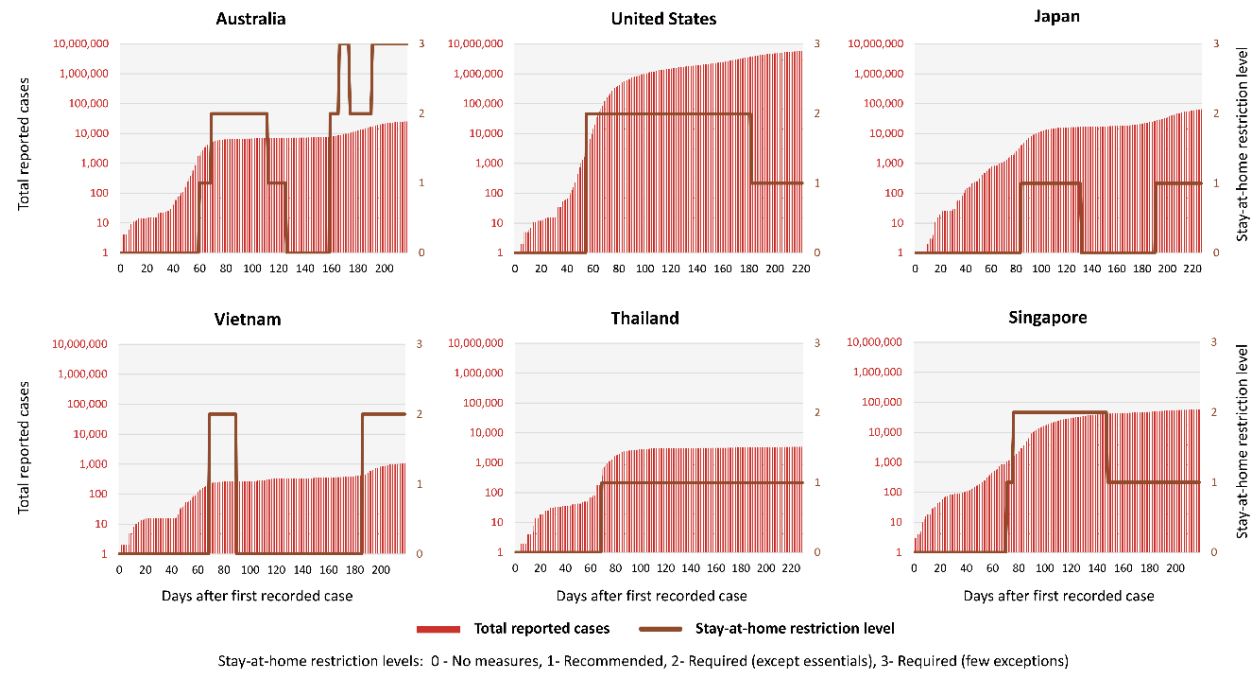

Stay-at-home restriction levels: 0 - No measures, 1- Recommended, 2-Required (except essentials), 3- Required (few exceptions)

\section{FIGURE 10.6}

Total reported cases vs stay-at-home restriction levels, days after first recorded case

As a consequence, our assumption that city designs that reflect extensive road infrastructure, public transit systems and high population densities might exacerbate the transmission of COVID-19, does not hold in its entirety. It appears, that only high population density is associated with COVID-19 cases although it highlights that the densely populated cities have reduced cases of COVID-19. From this cursory analysis, the threat posed by extensive transport systems and highly populated cities is less than first imagined and, is at least not as important factor as the public health orders deployed by city and government institutions to mitigate transmission of COVID-19.

\subsection{Conclusion}

As highlighted in the chapter, in the early stages of the pandemic, a comprehensive public health response including closing-down the transport systems in response to the rising cases of infection was necessary; this was certainly the situation in China. Similar responses were instituted across the globe albeit at varying time periods. In this chapter we explored, using mapping techniques, the role of city designs in the transmission of COVID-19 with the likelihood of cities with extensive public transit and high densities (described in this chapter as High Transit cities) having an increase propensity for transmission.

Despite being able to classify cities into distinct designs based on a combination of spatial characteristics and methods of artificial intelligence, when the city designs were assessed against the number of COVID-19 cases, the design of the city was not associated with the likely number of COVID-19 cases observed but rather high population densities and longer times to initiate stay-at-home orders reduced the COVID-19 cases observed.

What the findings in this chapter points to is that whatever the potential threats posed by cities with high population densities, extensive public transit or smaller block sizes with respect to the transmission of COVID-19, their effects are not dominant over the comprehensive implementation 
of social or public health interventions. This is not to say that living conditions in individual neighbourhoods, highly utilised public transit networks, or other city features might not provide circumstances for more ready transmission of communicable disease between individuals, but rather, that the evidence is not strong that city design is the primary driver. One alternate explanation to what is observed here is that what we are seeing reflects community resilience. Community resilience is the ability of communities/cities to respond positively to crises such as the pandemic. Countries/cities that responded comprehensively (and not necessarily promptly) to the threat of COVID-19 by instituting appropriate public health responses (as described in this chapter), see rapid declines in the rate of infections. This points to the role that good governance and community resilience can play when faced with emergencies such as a pandemic.

\section{References}

[1] Simiao Chen, Juntao Yang, Weizhong Yang, Chen Wang, and Till Bärnighausen. Covid-19 control in china during mass population movements at new year. The Lancet, 395(10226):764-766, Mar 2020. ISSN 0140-6736. doi: 10.1016/S0140-6736(20)30421-9. URL https://doi.org/10.1016/S0140-6736(20)30421-9.

[2] John Mathews. World war one's role in the worst ever flu pandemic. The Conversation, 2014. URL https://bit.1y/2S14Bn8. Accessed on 13 August 2020.

[3] Nature. 2020. URL https://go.nature.com/2S45NGj. Accessed on 13 August 2020.

[4] Huaiyu Tian, Yonghong Liu, Yidan Li, Chieh-Hsi Wu, Bin Chen, Moritz U. G. Kraemer, Bingying Li, Jun Cai, Bo Xu, Qiqi Yang, Ben Wang, Peng Yang, Yujun Cui, Yimeng Song, Pai Zheng, Quanyi Wang, Ottar N Bjornstad, Ruifu Yang, Bryan Grenfell, Oliver Pybus, and Christopher Dye. The impact of transmission control measures during the first 50 days of the covid-19 epidemic in china. medRxiv, 2020. doi: 10.1101/ 2020.01.30.20019844. URL https://www.medrxiv.org/content/early/2020/03/10/2020.01.30.20019844.

[5] Christopher A Mouton, Russell Hanson, Adam R Grissom, and John P Godges. COVID-19 Air Traffic Visualization: By January 31, 2020, at Least 1.5 Daily Infected Passengers Were Originating in China. RAND Corporation, 2020. URL https://www.rand.org/pubs/research_reports/RRA248-2.html.

[6] Lucy Budd and Stephen Ison. Responsible transport: A post-covid agenda for transport policy and practice. Transportation Research Interdisciplinary Perspectives, 6:100151, 2020. doi: 10.1016/j.trip.2020.100151.

[7] Our World in Data. 2020. URL https://bit.ly/3i8Arci. Accessed on 13 August 2020.

[8] Thomas Hale, Noam Angrist, Beatriz Kira, Anna Petherick, Toby Phillips, and Samuel Webster. Variation in government responses to covid-19. version 6.0. Blavatnik School of Government Working Paper, 2020. URL https://www.bsg.ox.ac.uk/covidtracke. Accessed on 14 August 2020.

[9] Annayath Maqbool and Noor Zaman Khan. Analyzing barriers for implementation of public health and social measures to prevent the transmission of covid-19 disease using dematel method. Diabetes $E_{S}$ Metabolic Syndrome: Clinical Research \&5 Reviews, 14(5):887-892, 2020. ISSN 1871-4021. doi: https://doi.org/10. 1016/j.dsx.2020.06.024.

[10] Jason Thompson, Mark Stevenson, Jasper S Wijnands, Kerry A Nice, Gideon Dpa Aschwanden, Jeremy Silver, Mark Nieuwenhuijsen, Peter Rayner, Robyn Schofield, Rohit Hariharan, et al. A global analysis of urban design types and road transport injury: an image processing study. The Lancet Planetary Health, 4 (1):e32-e42, 2020.

[11] United Nations, Department of Economic and Social Affairs, Population Division . World Urbanization Prospects: The 2014 Revision, Highlights (ST/ESA/SER.A/352). 2014.

[12] Christian Szegedy, Vincent Vanhoucke, Sergey Ioffe, Jon Shlens, and Zbigniew Wojna. Rethinking the inception architecture for computer vision. In Proceedings of the IEEE conference on computer vision and pattern recognition, pages 2818-26, 2016.

[13] Mark Stevenson, Jason Thompson, Thiago Hérick de Sá, Reid Ewing, Dinesh Mohan, Rod McClure, Ian Roberts, Geetam Tiwari, Billie Giles-Corti, Xiaoduan Sun, Mark Wallace, and James Woodcock. Land use, transport, and population health: estimating the health benefits of compact cities. The Lancet, 388(10062): 2925-2935, Dec 2016. ISSN 0140-6736. doi: 10.1016/S0140-6736(16)30067-8. 